\title{
Neural Compensation for Long-term Asymmetric Optical Blur to Improve Visual Performance in Keratoconic Eyes
}

\author{
Ramkumar Sabesan ${ }^{1}$ and Geunyoung Yoon ${ }^{2}$
}

Purpose. To investigate whether long-term visual experience with irregular optical blur compensates for the impact of higher-order aberration on visual performance in keratoconic (KC) eyes.

Methops. The aberrations and high (100\%)- and low (20\%)contrast tumbling E visual acuity (VA) were measured in four moderate $\mathrm{KC}$ eyes in which the subjects were wearing their own prescribed soft toric contact lenses over a 6-mm pupil. VA was measured in three emmetropic normal eyes for comparison with each of the four KC eyes. An adaptive optics system was used to correct the aberration of the normal eye and to induce the aberration of the $\mathrm{KC}$ eye simultaneously during vision testing. The magnitude of neural compensation was defined as improvement in VA in each KC eye compared with the normal eyes with $\mathrm{KC}$ aberrations.

REsults. Mean total and higher-order root mean square errors in the KC eyes with contact lenses were $2.72 \pm 0.83 \mu \mathrm{m}$ and $1.36 \pm 0.29 \mu \mathrm{m}$, respectively, for a $6-\mathrm{mm}$ pupil. Residual RMS wavefront error in induction of $\mathrm{KC}$ aberrations on normal eyes was approximately $0.1 \mu \mathrm{m}$ in all cases. Each $\mathrm{KC}$ eye had statistically better high $(P<0.02)$ - and low $(P<0.03)-$ contrast VA than the three normal eyes. Mean compensation for high-contrast VA in logMAR was $0.12 \pm 0.09$, corresponding to an improvement of $23.8 \%$. A similar result was obtained for low-contrast VA. The magnitude of compensation increased with the severity of KC aberrations.

Conclusions. In $\mathrm{KC}$ eyes, the neural visual system compensates for long-term visual experience with an asymmetrically blurred retinal image, resulting in improved visual performance. (Invest Ophthalmol Vis Sci. 2010;51:3835-3839) DOI:10.1167/ iovs.09-4558

T he retinal image quality of the human eye is degraded by lower- and higher-order aberrations. ${ }^{1-3}$ Higher-order aberrations, which remain after compensation of conventional refractive error, significantly deteriorate image quality. Recent technological advances in the field of ocular wavefront sensing and adaptive optics (AO) have added extensively to our understanding of ocular higher-order aberration and its impact on vision. ${ }^{4}$ Eyes with abnormal corneal conditions, such as those with keratoconus, are affected by remarkably larger magni-

From the ${ }^{1}$ Institute of Optics and the ${ }^{2}$ Flaum Eye Institute, University of Rochester, Rochester, New York.

Supported by National Institutes of Health/National Eye Institute Grant RO1 EY014999 and Research to Prevent Blindness.

Submitted for publication August 31, 2009; revised December 8, 2009; accepted January 24, 2010.

Disclosure: R. Sabesan, None; G. Yoon, None

Corresponding author: Ramkumar Sabesan, Flaum Eye Institute, G-3183A, 601 Elmwood Avenue, Box 314, Rochester, New York 14624; ramkumar@optics.rochester.edu. tudes of higher-order aberrations, approximately five to six times greater in terms of higher-order root mean square (RMS) wavefront error, than what is typically observed in normal eyes for a 6-mm pupil. ${ }^{5,6}$ Moreover, the dominant higher-order aberration in these eyes is negative vertical coma. Higher-order aberrations are left uncorrected with conventional correction with either soft or hard contact lens. Accordingly, even with their routine prescriptions, ketatoconic (KC) eyes experience asymmetric retinal image blur significantly greater than that found in normal eyes.

Correction of higher-order aberrations with AO to achieve significant visual benefit has been demonstrated. ${ }^{7,8}$ By surpassing the limit imposed by the optics of the eye, AO has facilitated the noninvasive investigation of the visual system in isolation. Recently, Sabesan and Yoon ${ }^{9}$ measured visual performance after correcting aberrations to nearly diffraction-limited optical quality in normal and highly aberrated $\mathrm{KC}$ eyes. Interestingly, although the retinal image quality was similar in both groups, visual acuity (VA) was significantly worse in KC eyes than in normal eyes. In addition, the magnitude of native higher-order aberrations before correction might have influenced the extent of this limitation of visual performance in these eyes. Such deficit in visual performance, when unaccounted for by optical theory, might be attributed in part to neural factors. The findings of Rossi et al. ${ }^{10}$ suggest similar neural factors limiting visual performance in low myopes compared with emmetropes after correction of all ocular aberrations.

Before the development of $\mathrm{AO}$ for vision testing, neural factors affecting spatial vision were studied by creating interference fringes directly on the retina after bypassing the ocular optics. ${ }^{11}$ Among many other applications, this technique was used to measure the neural response of subjects with ocular astigmatism to differently oriented gratings. ${ }^{12}$ Significant depression in neural contrast sensitivity was observed for the astigmatic meridian compared with the normal meridian. Such orientational neural deficit observed with laser interferometry in persons with astigmatism might be considered analogous to that observed in myopes and $\mathrm{KC}$ eyes after compensating for all higher-order aberrations. Visual experience with blurred retinal image quality induced by either lower- or higher-order aberrations might render the visual system insensitive to a diffraction-limited retinal image quality. Accordingly, the deficit is noted in the visual performance measured after $\mathrm{AO}$ correction. Furthermore, similar to neural insensitivity for a perfect retinal image, neural compensation for blurred retinal image may also be possible, thereby reducing its impact on visual performance. After extended periods of viewing through myopic blur, improvement in VA was reported that was not accompanied by a corresponding optical change. ${ }^{13,14}$ Webster et al. $^{15}$ further showed that adaptation to low-pass (blurred) filtered images resulted in the original image appearing "too sharp" and the best subjective focus shifting toward an image 


\section{$\mathrm{KC}$ aberration}

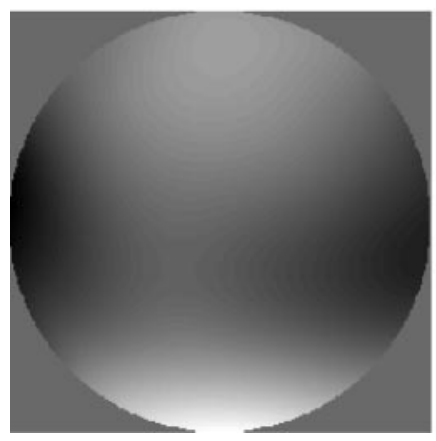

\section{$\mathrm{KC}$ aberration imposed} on normal eye

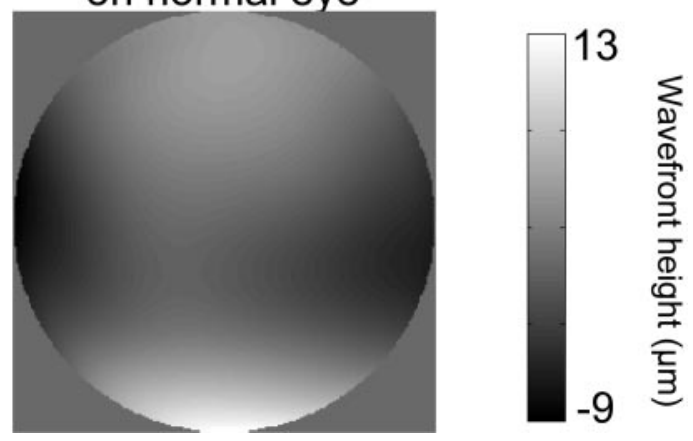

FIGURE 1. Wavefront map of a moderate $\mathrm{KC}$ eye with its routine prescription soft contact lens and of a normal eye that has the same aberration imposed on it. Both wavefront maps are on the same color bar. The residual error of aberration induction of was $0.09 \mu \mathrm{m}$ in RMS. with lower spatial frequencies. Adapting to aberrations through normal everyday viewing, unlike an induced adaptation to either refractive error or spatially filtered images, might lend more robustness to the phenomenon. In normal eyes, adaptation to optical blur induced through each subject's particular higher-order aberrations has been shown. ${ }^{16}$ Subjects preferred the blur induced by their own higher-order aberrations rather than a rotated version of the same. Such compensation effect might be even more remarkable in KC eyes, which are affected by significantly worse retinal image quality in everyday viewing, even with smaller daytime pupils. This study examined whether the neural visual system compensates for long-term visual experience with a blurred retinal image, resulting in improved visual performance. This was achieved by making emmetropic normal eyes optically identical to $\mathrm{KC}$ eyes through the use of $\mathrm{AO}$ and comparing their visual performance with that of $\mathrm{KC}$ eyes. Our hypothesis was that if $\mathrm{KC}$ eyes had adapted to their own aberrations, they would achieve better visual performance than normal eyes viewing through the same aberrated optics.

\section{Subjects ANd Methods}

The University of Rochester Research Review Board approved this research, and each subject signed an informed consent form before participation in this study. All procedures involving human subjects were conducted in accordance with the tenets of the Declaration of Helsinki. Measurements from four moderate $\mathrm{KC}$ eyes in which the subjects were wearing prescription soft toric contact lenses and measurements from three emmetropic normal eyes were used for this study. The soft toric contact lenses were the subjects' own everyday correction. Keratometric readings from corneal topography maps were used to classify the $\mathrm{KC}$ eyes as moderate, as suggested in the Collaborative Longitudinal Evaluation of Keratoconus study. ${ }^{17}$ Paralysis of accommodation and dilation of the pupil in all subjects were achieved with $1 \%$ tropicamide ophthalmic solution. Head movements were stabilized in all subjects using a bite bar mount. Mean total and higherorder RMS errors in the KC eyes with contact lenses were $2.72 \pm 0.83$ $\mu \mathrm{m}$ and $1.36 \pm 0.29 \mu \mathrm{m}$ for a $6-\mathrm{mm}$ pupil, respectively. Astigmatism and coma were the most dominant lower- and higher-order aberrations, respectively. The average higher-order RMS wavefront error was $0.40 \pm 0.21 \mu \mathrm{m}$ in the three emmetropically normal eyes over the same pupil size. The AO system used in this study consisted of a large-stroke deformable mirror ${ }^{18}$ (Mirao 52D; Imagine Eyes, Orsay, France) and a custom-made, in-house Shack-Hartmann wavefront sensor. The wave aberrations were calculated from the Shack-Hartmann spot array pattern by decomposing into 10th-order Zernike polynomials. The Zernike coefficients were expressed according to the ANSI Z80.28-2004 standard. ${ }^{19}$ VA was measured using a tumbling letter E test at high (100\%) and low (20\%) contrast in white light. The experimental apparatus and the psychophysical procedure for measuring VA have been described elsewhere. ${ }^{9,20}$ Subjects viewed the visual stimulus through an artificial adjustable pupil placed conjugate to the eye's pupil, which was always set to $6 \mathrm{~mm}$.

First, aberrations and high (100\%)- and low (20\%)-contrast tumbling E VA were measured in the four moderate $\mathrm{KC}$ eyes wearing soft toric contact lenses over a $6-\mathrm{mm}$ pupil. Subsequently, three emmetropic normal eyes were used to measure VA with the four KC eyes' aberrations imposed on them. For each KC eye, the same three emmetropic normal eyes were used to minimize intersubject variability. The AO system was used to correct the normal eyes' aberration and to induce the $\mathrm{KC}$ eye aberrations on them in a single concurrent step. This state of aberration was maintained dynamically throughout the VA test in a closed-loop manner to ensure stable optical quality. AO closed-loop gain of $90 \%$ to $100 \%$ ensured fast convergence within one to three iterations after blink. High closed-loop gain also facilitated a stable optical quality throughout the VA test. The subjects were asked to blink at their discretion during the psychophysical task, at which point the induction of KC aberration was temporarily suspended. In this manner, retinal image quality in the normal and KC eyes was equalized. Consequently, the role of neural factors in visual function could be compared between the two. The magnitude of neural compensation in each KC eye was defined as the improvement in its VA (logMAR) compared with the three normal eyes, on average, with the same aberrations.

\section{Results}

\section{Optical Performance of Inducing KC Aberrations on Normal Eyes}

Figure 1 shows the wavefront map of a moderate KC eye with its routine prescription soft contact lens and of a normal eye with the same aberration imposed on it. Both wavefront maps are on the same color bar. The residual error in induction of the aberrations was $0.09 \mu \mathrm{m}$ in this eye and near this value in all eyes. Figure 2 shows the representative time-course of RMS for one normal eye when the aberration of a moderate $\mathrm{KC}$ eye is imposed on it during the VA test. The dashed line indicates the measured RMS for the moderate KC eye. Peaks in the time-course correspond to blinks during the VA test, at which points the induction was suspended. The aberrations were imposed reliably, and residual error was maintained around 0.1 $\mu \mathrm{m}$ throughout the VA test. It is important to note here that the correction of normal eye aberration and the induction of a $\mathrm{KC}$ eye aberration are performed concomitantly. Averaged variability of aberration induction, defined as the standard deviation of residual RMS during acuity test, was approximately $0.02 \mu \mathrm{m}$ for all normal eyes. Thus, using AO, the same aberrated optical quality found habitually in $\mathrm{KC}$ eyes was reliably provided to normal eyes. In addition, the ability to maintain this optical 




FIGURE 2. Time-course of RMS for one normal eye when a moderate $\mathrm{KC}$ eye's aberration is imposed on it during VA testing. Curve: evolution of RMS with time. At approximately 6.5 seconds, the $\mathrm{KC}$ aberration is imposed. Dashed line: RMS for the moderate KC eye. Peaks in the time-course correspond to blinks during the VA test, at which points correction was suspended.

quality accurately for a relatively long period facilitated consistent vision testing.

\section{Visual Acuity in $\mathrm{KC}$ and Normal Eyes with the Same KC Aberration}

Figure 3 shows the comparison of visual performance between $\mathrm{KC}$ eyes and normal eyes with KC aberration. The $x$-axis shows the VA (logMAR) in KC eyes when viewing through everyday prescription soft contact lenses, and the $y$-axis shows the VA (logMAR) in normal eyes with KC aberration. Circles and squares denote high- and low-contrast VA, respectively. Each data point represents the average of three normal eyes for each $\mathrm{KC}$ eye. The dashed line is the equal acuity line; data points lying above the line indicate better VA in $\mathrm{KC}$ eyes with their

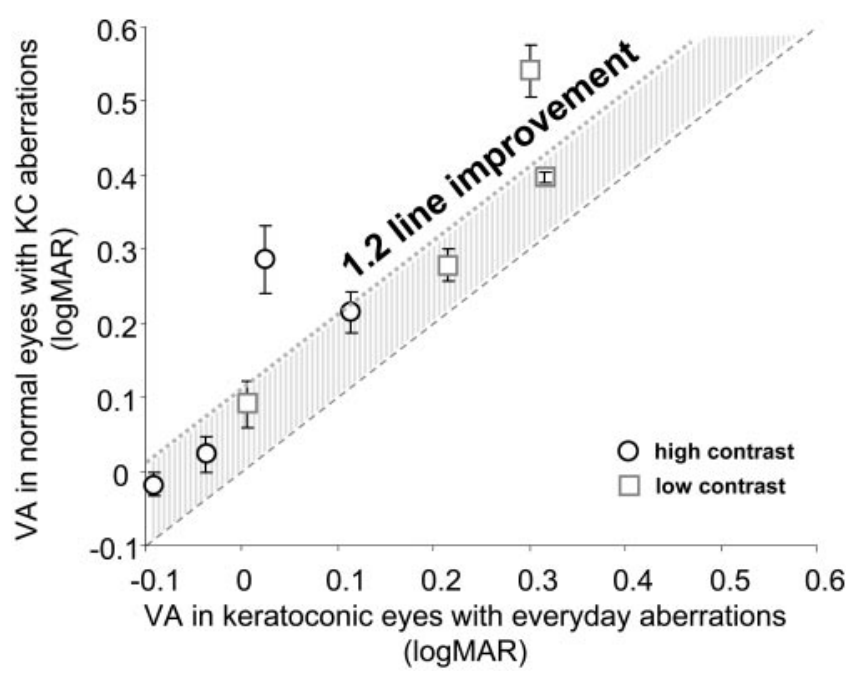

Figure 3. Comparison of visual performance between $\mathrm{KC}$ eyes and normal eyes with $\mathrm{KC}$ aberrations. Each data point represents the average of three normal eyes for each $\mathrm{KC}$ eye. Dashed line: equal acuity line; shaded region: average 1.2-line improvement in VA in KC eyes.

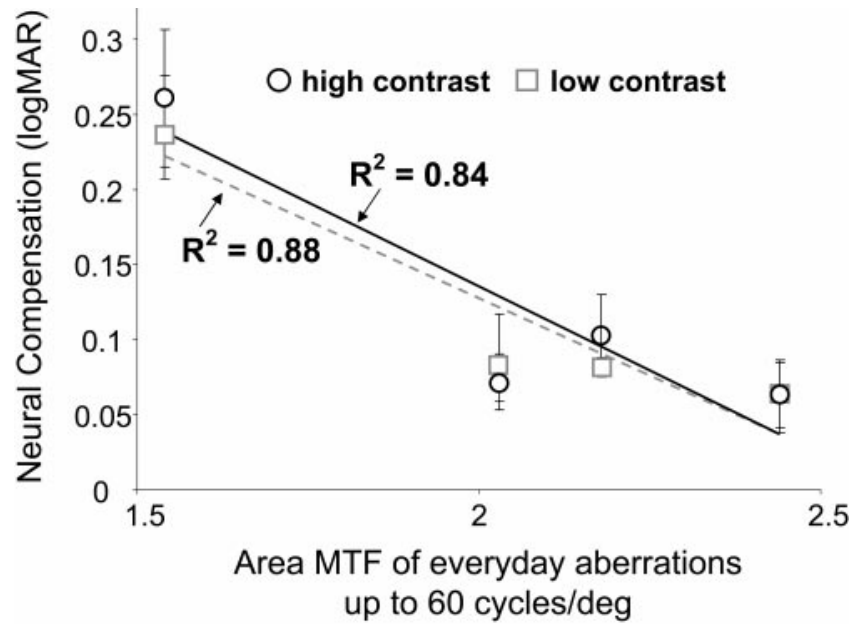

FIGURE 4. Neural compensation (logMAR) versus theoretical everyday retinal image quality. Retinal image quality is represented as the area under the MTF calculated up to $60 \mathrm{cyc} / \mathrm{deg}$. The coefficient of correlation for a linear fit for high- and low-contrast neural compensation is also indicated.

own everyday aberration and vice versa. KC eyes had significantly better high $(P<0.02)$ and low $(P<0.03)$ contrast VA than did normal eyes. With equivalent optical quality, neural compensation accounted for an average 1.2-line improvement in both high- and low-contrast VA in the $\mathrm{KC}$ eyes compared with the normal eyes.

Figure 4 illustrates the correlation between the neural compensation (logMAR) and everyday retinal image quality in the KC eyes. Retinal image quality is represented on the $x$-axis as the area under the modulation transfer function up to 60 cycles per degree, calculated from the everyday aberrations in $\mathrm{KC}$ eyes. Each data point again represents the average of three normal eyes for each KC eye. KC eyes with poorer everyday retinal image quality demonstrated greater magnitude of neural compensation. While estimating retinal image quality with regard to area under the modulation transfer function, a wellcorrelated trend of decreasing neural compensation was observed with an improvement in optical quality. The correlation was similar for both high- and low-contrast neural compensation. Neural compensation thus depends on the severity of native optical quality: poorer retinal image quality necessitates larger magnitude of neural compensation and vice versa.

\section{Discussion}

Spatial vision is governed by both optical and neural factors. To understand how long-term visual experience with irregular optical blur affects spatial vision, optical quality was equalized between normal and $\mathrm{KC}$ eyes using $\mathrm{AO}$. This facilitated an isolated investigation of the neural visual system alone in $\mathrm{KC}$ eyes. With equivalently blurred image quality, $\mathrm{KC}$ eyes achieved significantly better VA than normal eyes. This might suggest that the visual system is capable of compensating for asymmetrically blurred retinal image quality because of significant magnitudes of higher-order aberrations, resulting in improved visual performance in $\mathrm{KC}$ eyes.

It has been shown previously that adaptation to retinal image blurred by symmetric refractive error can lead to improved VA. Mon-Williams et al. ${ }^{13}$ noted an improvement in monocular VA of $0.12 \operatorname{logMAR}$ after 30 minutes of viewing through a $+1.00-\mathrm{D}$ lens without any significant change in the refractive state of the eye. Coincidentally, the improvement in VA in our study was similar to that noted by Mon-Williams et 
al. ${ }^{13}$ However, natural daytime pupils were used for both adaptation to defocus and measurement of VA in their study. In our study, despite the relatively large magnitudes of aberrations in KC eyes, a substantial 1.2-line improvement in VA was noted. Interestingly, the improvement was correlated with the severity of everyday retinal image quality and thus the adaptation state of the eye. The $\mathrm{KC}$ eye with maximum compensation might, however, skew the regression curves in Figure 4. On excluding this $\mathrm{KC}$ eye, the negative slopes of the regression lines for high- and low-contrast compensation were reduced by $79 \%$ and $73 \%$, respectively. More subjects must be studied to establish reliable statistical significance. Similarly, George and Rosenfield ${ }^{14}$ demonstrated that the mean change in grating VA was significantly greater for myopic subjects than for emmetropic subjects while viewing through $+2.50 \mathrm{D}$ lens for 120 minutes and concluded that the neural compensation was dependent on the refractive state of the eye. It is, however, difficult to make a direct comparison between these studies on adaptation to refractive error and this study on $\mathrm{KC}$ eyes. The magnitude of the compensation might be higher in $\mathrm{KC}$ eyes adapting to their own aberrations through normal everyday viewing, unlike a synthetically induced adaptation to either refractive error or spatially filtered images. ${ }^{15}$ The rate at which the plastic visual system adapts to its monochromatic aberrations is still unknown. Nevertheless, an improvement in spatial vision after the long-term visual experience with highly aberrated optics, unlike after a temporarily induced adaptation, might denote an approximate upper limit to the compensatory time-course of the visual system.

In their study, Mon-Williams et al. ${ }^{13}$ suggested that a possible reason for improvement in visual resolution was the unmasking of high spatial frequency selective channels while balancing the channel gains in response to the spatial frequency distribution of the retinal image. An improvement in visual resolution, as found in $\mathrm{KC}$ eyes, might also be as a result of such a mechanism given the relatively long visual experience with the shape of the retinal image blur. In everyday viewing with conventional correction, either in high- or lowlight conditions or when accommodating, $\mathrm{KC}$ eyes experience significant blur. With conventional correction, the residual coma in $\mathrm{KC}$ eyes gives rise to an orientation-specific blur, unlike the rotationally symmetric blur introduced by defocus. Neurons in the visual system are known to be selectively sensitive to orientation and spatial frequency of retinal images. ${ }^{21}$ Accordingly, the neural compensatory mechanism in $\mathrm{KC}$ eyes is also likely to be mediated by orientation-sensitive sites of the visual cortex.

In addition to an improvement in threshold spatial resolution, an enhancement in suprathreshold spatial vision might be expected in $\mathrm{KC}$ eyes after the long-term visual experience with aberrated retinal image quality. The visual system might recognize the amplitude spectrum of retinal images and develop a rule by which the contrast gains of spatial frequency channels are adjusted so as to optimize visual performance. This phenomenon of contrast gain adjustment is referred to as contrast constancy, ${ }^{22}$ analogous to other constancies in vision. To investigate the presence of contrast compensation, we measured suprathreshold contrast matching in KC eyes (manuscript in preparation). Contrast constancy defines the normal operating characteristic for suprathreshold vision and has been studied in cases of visual function loss. ${ }^{23,24}$ The visual diet of highly aberrated $\mathrm{KC}$ eyes is dominated by lower frequencies; therefore, the suprathreshold contrast compensation might also be most prevalent at these frequencies.

Aberrations in the eye are dynamic in nature ${ }^{25}$ and change with pupil diameter, accommodation, age, and tear film dynamics. In both high- and low-light conditions, KC eyes experience significant blur because of the magnitude of higher- order aberrations. However, the shape of the point-spread function might be different to only within a scaling factor ${ }^{9}$ with varying pupil sizes. Moreover, there is some evidence that the visual system is known to become accustomed to more than one point-spread function given sufficient experience with each. ${ }^{26}$ Nevertheless, it is important to investigate the influence of pupil size on neural compensation.

We have demonstrated that $\mathrm{KC}$ eyes show evidence of a neural compensatory mechanism to alleviate the effect of higherorder aberrations on visual performance. However, aberrated optics still impose the ultimate limit to visual performance in these eyes. After correcting aberrations to near diffractionlimited optical quality, Sabesan and Yoon' recently demonstrated a poorer visual performance in $\mathrm{KC}$ eyes compared with normal eyes, attributing the deficit to neural factors. Chronic exposure to aberrated retinal images, on the other hand, might render the visual system insensitive to finer spatial detail. Analogous to adaptation to aberrated image quality, an interesting and clinically relevant question to address is whether the visual system can be readapted to near diffraction-limited ocular optics. Provision of superior optical quality noninvasively for a longer duration is the primary challenge in investigating this question. Nevertheless, with customized vision correction methods rapidly gaining importance for compensating for higher-order aberrations and presbyopia, the importance of neural factors governing visual performance cannot be overemphasized.

\section{References}

1. Smirnov MS. Measurement of the wave aberration of the human eye. Biophysics. 1962;7:766-795.

2. Porter J, Guirao A, Cox IG, Williams DR. Monochromatic aberrations of the human eye in a large population. $J$ Opt Soc Am A. 2001;18:1793-1803.

3. Thibos L, Hong X, Bradley A, Cheng X. Statistical variation of aberration structure and image quality in a normal population of healthy eyes. J Opt Soc Am A. 2002;19:2329-2348.

4. Liang J, Williams DR. Aberrations and retinal image quality of the normal human eye. J Opt Soc Am A. 1997;14:2873-2883.

5. Maeda N, Fujikado $\mathrm{T}$, Kuroda $\mathrm{T}$, et al. Wavefront aberrations measured with Hartmann-Shack sensor in patients with keratoconus. Opbthalmology. 2002;109:1996-2003.

6. Pantanelli S, MacRae S, Jeong T, Yoon G. Characterizing the wave aberration in eyes with keratoconus or penetrating keratoplasty using a high-dynamic range wavefront sensor. Ophthalmology. 2007; 114:2013-2021.

7. Liang J, Williams DR, Miller D. Supernormal vision and highresolution retinal imaging through adaptive optics. J Opt Soc Am A. 1997; 14:2882-2892.

8. Yoon GY, Williams DR. Visual performance after correcting the monochromatic and chromatic aberrations of the eye. $J$ Opt Soc Am A. 2002;19:266-275.

9. Sabesan R, Yoon G. Visual performance after correcting higher order aberrations in keratoconic eyes. J Vis. 2009;9:1-10.

10. Rossi E, Weiser P, Tarrant J, Roorda A. Visual performance in emmetropia and low myopia after correction of high-order aberrations. J Vis. 2007;7:14.

11. Campbell FW, Green DG. Optical and retinal factors affecting visual resolution. J Pbysiol (Lond). 1965;181:576-593.

12. Freedman R, Thibos L. Contrast sensitivity in humans with abnormal visual experience. J Physiol. 1975;247:687-710.

13. Mon-Williams M, Tresilian J, Strang N, Kochhar P, Wann J. Improving vision: neural compensation for optical defocus. Proc Biol Sci. 1998;265:71-77.

14. George S, Rosenfield M. Blur adaptation and myopia. Optom Vis Sci. 2004;81:543-547.

15. Webster M, Georgeson M, Webster S. Neural adjustments to image blur. Nat Neurosci. 2002;5:839-840. 
16. Artal P, Chen L, Fernández E, Singer B, Manzanera S, Williams D Neural compensation for the eye s optical aberrations. J Vis. 2004; 4:281-287.

17. Zadnik K, Barr J, Gordon M, Edrington T. Biomicroscopic signs and disease severity in keratoconus: Collaborative Longitudinal Evaluation of Keratoconus (CLEK) Study Group. Cornea. 1996;15:139-146.

18. Fernandez E, Vabre L, Hermann B, Unterhuber A, Povazay B, Drexler W. Adaptive optics with a magnetic deformable mirror: applications in the human eye. Opt Expr. 2006;14:8900-8917.

19. Thibos LN Applegate RA, Schwiegerling JT, Webb R, VSIA Standards Taskforce Members. Standards for reporting the optical aberrations of eyes: OSA trends in optics and photonics. In: Lakshminarayanan V, ed. Vision Science and Its Applications. Vol. 35. Washington, DC: Optical Society of America; 2000:232-244.

20. Sabesan R. Correcting highly aberrated eyes using large-stroke adaptive optics. J Refract Surg. 2007;23:947-952.
21. Blakemore C, Campbell FW. On the existence of neurones in the human visual system selectively sensitive to the orientation and size of retinal images. J Physiol (Lond). 1969;203:237-260.

22. Georgeson MA, Sullivan GD. Contrast constancy: deblurring in human vision by spatial frequency channels. J Physiol (Lond). 1975;253:627-656.

23. Mei M, Leat S. Suprathreshold contrast matching in maculopathy. Invest Ophthalmol Vis Sci. 2007;48:3419-3424.

24. Delahunt P, Hardy J, Okajima K, Werner J. Senescence of spatial chromatic contrast sensitivity, II: matching under natural viewing conditions. J Opt Soc Am A. 2005;22:60 - 67.

25. Hofer H, Artal P, Singer B, Aragon JL, Williams DR. Dynamics of the eye's wave aberration. J Opt Soc Am A. 2001;18:497-506.

26. Peterson J, Peterson JK. Does practice with inverting lenses make vision normal. Psychol Monographs. 1938;50:12-37. 